\title{
A CAMERA-BASED MEASUREMENT SYSTEM FOR OPTIMIZATION OF DIFFERENTIAL-ALGEBRAIC ECOTOXICOLOGICAL MODELS
}

\author{
Pawel Drąg, ${ }^{1}$ Krystyn Styczeń, ${ }^{2}$ Konrad Matyja ${ }^{3}$
}

\begin{abstract}
We present a general framework for measurements and optimization of differential-algebraic models. Moreover, we propose an application of the considered methodology in ecotoxicology. The differential-algebraic models can be used to describe different ecotoxicological relations. One of them is the influence of the environmental pollution on the Daphnia's movement characteristics. Changes in these characteristics can be used as a tool for assessment of neurotoxicity. The camerabased measurement and optimization system enable us to obtain the differential-algebraic ecotoxicological relations in a fully automated way.
\end{abstract}

UDC Classification: 519.6; DOI: http://dx.doi.org/10.12955/cbup.v5.1074

Keywords: differential-algebraic models, ecotoxicological models, measurement system, nonlinear optimization

\section{Introduction}

The aim of this study is to present and discuss a new method for measurement, which uses a camerabased observation system. The general difficulty in the presented methodology is connected with an appropriate interpretation of the obtained measured values and an optimization of parameters of a model. The considered task has an important practical application in the modeling of ecotoxicological phenomena. Moreover, the presented approach summarizes and extends the recently obtained results.

A fast development of new industrial technologies can generate a significant amount of pollution. Especially xenobiotic substances from technological processes, which can be introduced into the environment, can be dangerous for living organisms. Therefore, the toxicity of these substances has to be assessed to predict the possible impact on ecosystems.

A general idea of a toxicity assessment consists of determining the relationship between a dose (or concentration) and the toxic effects, which can be divided into two main groups:

- discrete effects

- mortality, i.e. the number of immobilized organisms,

- continuous effects

- changes in enzyme activity,

$\circ$ the rate of growth.

In practice, national and international institutions and organizations, like US Environmental Protection Agency (US EPA), Organization for Economic Co-operation and Development (OECD), International Organization for Standardization (ISO), often normalize toxicity tests. Documents provided by them contain a detailed description of conditions, as well as the methodology of required experiments.

Unfortunately, the toxicity assessment methods typically possess some disadvantages.

1. Changes of toxic effects during the period of exposure are often ignored (Hatano \& Shoji, 2010).

2. Measurements are carried out with arbitrarily selected exposure time (Matyja et al. 2016).

3. Some effects, like mortality, provide a very little information about properties of the toxins.

4. Other effects require long-term measurements for their analysis.

Therefore, there is a request for a possible simple toxicity test, which would eliminate most of the mentioned disadvantages.

In this article, we considered an idea of a pattern motion analysis as a new toxicity test, that would possess the desired properties. Some chemical substances can affect the behavior of an observed organism. The nervous system, as well as the movement abilities, can be impaired. An impact of chemical pollutants can be observed and analyzed clearly and in depth on the example of Daphnia

\footnotetext{
${ }^{1}$ Wrocław University of Science and Technology, Wrocław, Poland. E-mail: pawel.drag@pwr.edu.pl

${ }^{2}$ Wrocław University of Science and Technology, Wrocław, Poland. E-mail: krystyn.styczen@pwr.edu.pl

${ }^{3}$ Faculty of Chemistry, Wrocław University of Science and Technology, Poland. Email: konrad.matyja@ pwr.edu.pl
} 
magna and crab Barytelphusa Guerini (Guilhermino et al., 1996; Fingerman et al., 1996). In particular, insecticides parathion and paraoxon can inhibit acetylcholinesterase of Daphnia magna (Guilhermino et al., 1996). Some substances, e.g., glucose and caffeine, may increase the mobility in general. Conversely, other substances can decrease the mobility. Moreover, the analysis of mobility can be applied not only to a single organism but also to groups, herds, and shoals. Some compelling studies regarding this matter have been conducted (Mach \& Schweitzer, 2007; Ordemann et al., 2003).

In the presented study, an application of motion pattern analysis of a single Daphnia magna has been considered. The observed motion changes can be used to illustrate the influence of some chemicals on Daphnia. Moreover, they can be used to assess their toxicity. The camera vision systems play a key role in many processes. In the discussed issue, using camera observations is required by a high frequency of changes, as well as by the size of the object observed, which is not larger than several millimeters. Long-term observations can be very tiring for the human eye, and this may lead to inaccurate results of the measurements.

The continuously operating vision systems are employed in many branches of environmental engineering. They can be used for direct observations of the essential parameters, as well as in the indirect observations, where the appropriate indicators are taken into account. Therefore, the main application of the proposed camera vision system is to find the relation between the indicators of pollution indicators and the observed behavior of the object.

The relations between the elements of the studied system can be modeled in many ways (Ahlkrona et al., 2016). One of the most commonly used ways is to describe the relations by ordinary differential equations (ODEs) (Balsa-Canto et al., 2005). In some cases, when not only differential but also algebraic relations can be defined, the differential-algebraic equations (DAEs) are employed. The algebraic relations, above all, express physical conservation laws of physical quantities such as mass, energy or momentum. Moreover, the algebraic equations can represent the geometrical constraints (Brenan et al., 1996; Diehl et al., 2002).

Therefore, the aim of this project was to design a camera-based vision system, which can observe the organism and model its motion, as well as to identify parameters of the unknown model (Betts, 2010; Biegler, 2010). The position changes of Daphnia magna have been described using new systems of differential-algebraic equation (DAEs). Subsequently, the nonlinear optimization algorithm was applied to identify the unknown model parameters.

Three milestones had to be achieved to realize the presented task. The required and available materials needed to be specified. Then, features of the motion patterns had to be combined into the new differential-algebraic model. Finally, the nonlinear optimization approach was proposed to evaluate the unknown model parameters.

\section{Materials}

Daphnia magna is small crustacean living in the freshwater environment. Its maximal length is $5 \mathrm{~mm}$. It is widely used for ecotoxicology purposes (El-Doma, 2013). Daphnis motion is very characteristic. They move by a series of "jumps," therefore is similar to flea movement (Daphnis are often called "water fleas"). The body of Daphnia is shown in Figure 1.

\begin{tabular}{|l|}
\hline Figure 1: Daphnia magna. \\
\hline \\
\hline Source: Author \\
\hline
\end{tabular}




\section{The Measurement System}

The main ideas of a fully automated vision system include data storage units, as well as imageprocessing algorithms for solving such problems as localization and path following of the objects observed. In recent years, the considered vision systems have been introduced in ecology, ecotoxicology, and environmental and agriculture engineering. For improvement of the efficiency and flexibility of such systems, the data could be obtained from different vision-based sensors, and the data-structure should be suitable for advanced image processing algorithms as well. Therefore, the required information can be obtained using the implemented reliable numerical procedures.

As it was indicated above, automated vision systems require both vision sensors, as well as image processing algorithm to obtain useful information about the systems, their quality, and other features. The environment can be characterized by lots of parameters, which are dependent on the available sensors and data acquisition possibilities.

The main part of the designed measurement system is the high-speed camera Optronis CL600x2 (Figure 2). The particular features of the camera, important from the application in measurement system point of view, have been presented in Table 2.

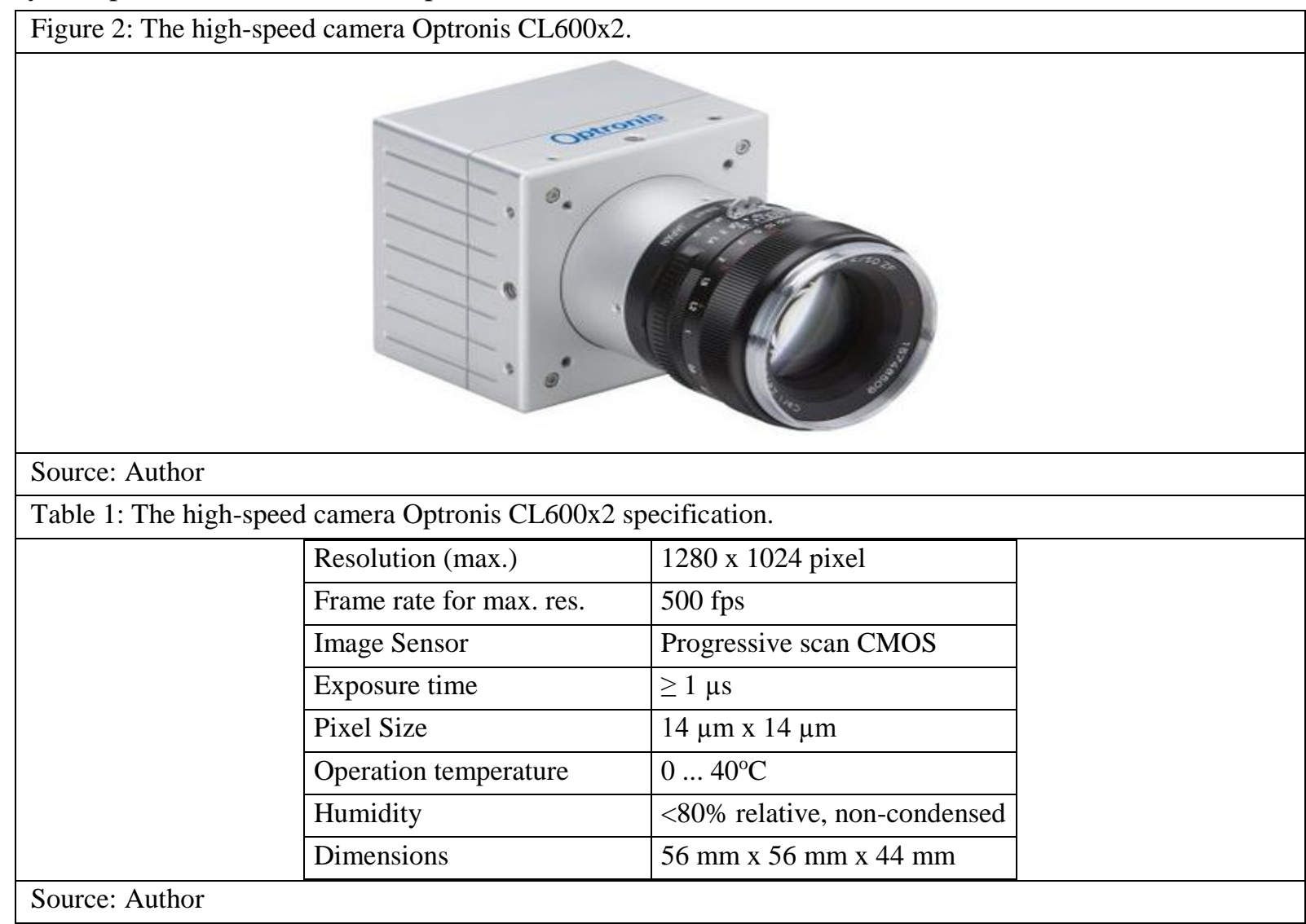

\section{The image processing algorithm}

The most prominent part of Daphnia are its eyes. For the identification of the eye position on an image, a threshold approach is usually sufficient. Therefore, a light background is necessary to avoid any inaccuracies.

The position of Daphnia can be equated with a position of their eyes. Therefore, only two coordinates are needed to define a position of an observed organism.

The position of Daphnia at time $t=t_{i} \quad$ can be defined as

$$
S_{i}=\left(x_{i}, y_{i}\right) \text {, }
$$

where $S_{i}$ denotes a state at time $t_{i}$, which can be determined by values of $x$ and $y$-axis: $x_{i}$ and $y_{i}$, respectively. The state at time $t=t_{i+1}$ can be defined similarly as

$$
S_{i+1}=\left(x_{i+1}, y_{i+1}\right)
$$


and in general

$$
M_{i \rightarrow i+1}=\left(\Delta x_{i \rightarrow i+1}, \Delta y_{i \rightarrow i+1}\right),
$$

where $M_{i \rightarrow i+1}$ denotes a movement from the state at time $t_{i}$ to the state at time $t_{i+1}$. Therefore

$$
S_{i}+M_{i \rightarrow i+1}=\left(x_{i}, y_{i}\right)+\left(\Delta x_{i \rightarrow i+1}, \Delta y_{i \rightarrow i+1}\right)=\left(x_{i+1}, y_{i+1}\right)=S_{i+1} .
$$

The sequence of the observed movements can take a form of the series

$$
\left\{M_{i \rightarrow i+1}\right\}_{i=0}^{N-1}=\left\{M_{0 \rightarrow 1}, M_{1 \rightarrow 2}, \cdots, M_{N-1 \rightarrow N}\right\},
$$

where $N$, the number of steps, does not need to be known a priori.

\section{The new differential-algebraic model}

The presented considerations lead us to the new form of the Daphnia's motion model. Each observed step

$$
S_{i+1}-S_{i}=M_{i \rightarrow i+1}
$$

has been taken at a known time. Therefore, the position change can be defined as

$$
\frac{S_{i+1}-S_{i}}{t_{i+1}-t_{i}}=\frac{M_{i \rightarrow i+1}}{t_{i+1}-t_{i}} .
$$

If the interval $t_{i+1}-t_{i}$ is constant, then

$$
\frac{S_{i+1}-S_{i}}{\Delta t}=\frac{M_{i \rightarrow i+1}}{\Delta t}
$$

Moreover, the differential motion model can be obtained

$$
\dot{S}=M_{\dot{S}}=\left[\begin{array}{l}
M_{\dot{x}} \\
M_{\dot{y}}
\end{array}\right]=\left[\begin{array}{l}
\dot{x} \\
\dot{y}
\end{array}\right]
$$

with the initial conditions

$$
\left[\begin{array}{l}
x\left(t_{0}\right) \\
y\left(t_{0}\right)
\end{array}\right]=\left[\begin{array}{l}
x_{0} \\
y_{0}
\end{array}\right],
$$

which are known from the measurements. The values $M_{\dot{x}}$ and $M_{\dot{y}}$ are unknown and needed to be estimated. Moreover, the geometrical constraints have to be incorporated into the model. These are the extreme values of the state coordinates

$$
x_{\min }<x(t)<x_{\max }
$$

and

$$
y_{\text {min }}<y(t)<y_{\text {max }}
$$

In this way, the new model of the Daphnia's motion with the differential-algebraic constraints has been obtained.

\section{The nonlinear optimization approach}

The data obtained from measurements $\left\{\left(x_{\text {meas }}\left(t_{i}\right), y_{\text {meas }}\left(t_{i}\right)\right)\right\}_{i=1}^{N}$ enable us to design the following nonlinear optimization task

$$
\sum_{i=1}^{N}\left(x_{\text {meas }}\left(t_{i}\right)-x\left(t_{i}\right)\right)^{2}+\left(y_{\text {meas }}\left(t_{i}\right)-y\left(t_{i}\right)\right)^{2} \rightarrow \min
$$

with the differential-algebraic continuous-pointwise constraints

$$
\begin{aligned}
& \dot{x}\left(t_{i}\right)=M_{\dot{x}} \\
& \dot{y}\left(t_{i}\right)=M_{\dot{y}} \\
& x\left(t_{0}\right)=x_{0} \\
& y\left(t_{0}\right)=y_{0}
\end{aligned}
$$




$$
\begin{aligned}
& x_{\text {min }}<x(t)<x_{\text {max }} \\
& y_{\text {min }}<y(t)<y_{\text {max }}
\end{aligned}
$$

for $t \in\left[\begin{array}{ll}t_{0} & t_{N}\end{array}\right]$.

In the proposed methodology, the successive quadratic optimization algorithm has been suggested (Nocedal \& Wright, 2006). Moreover, the optimization can be carried out simultaneously with the measurements. The results of the optimization from the previous iteration can be treated as a new starting point for the optimization with the extended set of measurements. This approach is appropriate for a huge set of measured data.

\section{Conclusions}

The main contribution of the presented work is the camera-based observation system of Daphnia's mobility. The general system consists of the measurement, as well as the algorithm for model parameters estimation.

The process of data acquisition and data analysis can be fully automated through the use of cameras and Matlab software. This kind of automation is a considerable advantage, which may result in shortening time of analysis of a large samples number.

Moreover, the new model of Daphnia's mobility consisting of the piecewise-continuous differentialalgebraic constraints has been introduced. The presented methodology can found its application in ecology and environmental engineering, where the Daphnia are employed as the indicators of environmental pollution.

\section{Acknowledgments}

The work of Paweł Drąg and Krystyn Styczen has been supported by the National Science Center under grant: DEC-2012/07/B/ST7/01216.

\section{References}

Ahlkrona, J., Lötstedt, P., Kirchner, N., and Zwinger T,. Dynamically coupling the non-linear Stokes equations with the Shallow Ice Approximation in glaciology: Description and first applications of the ISCAL method. Journal of Computational Physics, 2016, 308:1-19.

Balsa-Canto, E., Vassiliadis, V.S., and Banga, J.R. Dynamic Optimization of Single- and Multi-Stage Systems Using a Hybrid Stochastic-Deterministic Method. Ind. Eng. Chem. Res., 2005, 44:1514-1523.

Betts, J.T. Practical Methods for Optimal Control and Estimation Using Nonlinear Programming. Second Edition. SIAM, Philadelphia, 2010.

Biegler, L.T. Nonlinear Programming. Concepts, Algorithms and Applications to Chemical Processes. SIAM, Philadelphia, 2010 .

Brenan, K.E., Campbell, S.L., and Petzold, L.R. Numerical Solution of Initial-Value Problems in Differential Algebraic Equations. SIAM, Philadelphia, 1996.

Diehl, M., Bock, H.G., Schlöder, J.P., Findeisen, R., Nagy, Z., and Allgöwer, F. Real-time optimization and nonlinear model predictive control of processes governed by differential-algebraic equations. Journal of Process Control, 2002, 12:577-585.

El-Doma, M.O. Daphnia: Biology and Mathematics Perspectives. Nova Science Pub Inc, 2013.

Fingerman, M., Devi, M., Reddy, P.S., Katyayani, R. Impact of Heavy Metal Exposure on the Nervous System and Endocrine-Mediated Processes in Crustaceans. Zoological Studies, 1996, 35:1-8.

Guilhermino, L., Lopes, M.C., Carvalho, A.P., and Soares, A.M.V.M. Inhibition Of Acetylcholinesterase Activity As Effect Criterion In Acute Tests With Juvenile Daphnia magna. Chemosphere, 1996, 32:721-738.

Hatano, A. and Shoji, R. A new model for predicting time course toxicity of heavy metals based on Biotic Ligand Model (BLM). Comparative Biochemistry and Physiology Part C: Toxicology and Pharmacology, 2010, 151:25-32.

Mach, R. and Schweitzer F. Modeling Vortex Swarming In Daphnia. Bulletin of Mathematical Biology, 2007, 69:539-562.

Matyja, K., Małachowska-Jutsz, A., Mazur, A.K. and Grabas, K. Assessment of toxicity using dehydrogenases activity and mathematical modeling. Ecotoxicology, 2016, 25:924-939.

Nocedal, J. and Wright, S.J. Numerical Optimization. Springer, New York, 2006.

Optronis GmbH: Make time visible. CamRecord CL600x2 High Speed Camera, http://www.optronis.com/fileadmin/Upload/Product/CL/CL600x2_engl.pdf.

Ordemann, A., Balazsi, G., and Moss, F. Pattern formation and stochastic motion of the zooplankton Daphnia in a light field. Physica A: Statistical Mechanics and its Applications, 2003, 325:260-266. 